\title{
Mef2 in sickness and in health
}

Infection can cause weight loss, but the link between infection-induced immune activation and loss of anabolic function is poorly understood. Now, researchers studying infection in Drosophila melanogaster have found that the transcription factor Mef2 (myocyte-specific

Mef2 ... controls a switch from metabolic gene expression to immuneresponse gene expression during infection in flies
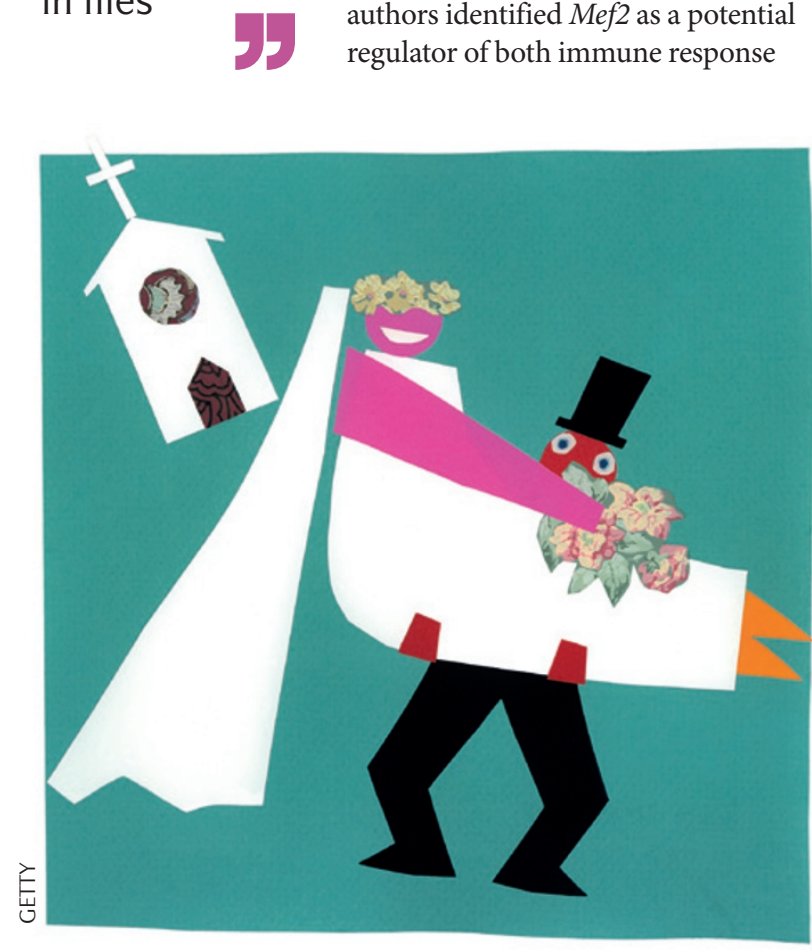

genes and metabolic activities. In the functional RNAi screens, Mef2 was one of five genes that were required for D. melanogaster survival following Mycobacterium marinum infection. Moreover, flies with a targeted knockdown of Mef2 expression in the fat body (which is equivalent to the mammalian liver and adipose tissue) showed increased susceptibility to infection with several other microorganisms (Listeria monocytogenes, Enterobacter cloacae and Candida albicans), which target the Toll or Imd pathways.

Further analysis of the microarray data revealed that a cluster of genes predicted to be targeted by Mef 2 contained metabolic enzymes that were rapidly and transiently downregulated 3-6 hours after infection. Similarly, the expression of a group of enzymes that are involved in glycogenesis and lipogenesis was lower in Mef2-knockdown D. melanogaster than in control flies, and Mef2-knockdown flies died more rapidly when starved. This suggests that a metabolic defect in Mef2-knockdown flies contributes to their poor survival after infection by decreasing their ability to withstand infection-induced weight loss.

In addition to this metabolic defect, Mef2-knockdown D. melanogaster showed a marked reduction in their expression of various antimicrobial peptides (AMPs) in response to infection. Analysis of putative regulatory regions from AMP genes consistently revealed the presence of a predicted Mef2-binding site that overlapped with a TATA box (which is bound by TATA-binding protein (Tbp)). This Mef2-TATA box-binding site was shown to be required for the induction of AMP expression in vivo using flies expressing wild-type or mutant reporter constructs. Further experiments indicated that Mef2 and Tbp form a complex on the Mef2-TATA boxbinding sites of numerous AMPs, as well as of other immune genes, and that this is required for the normal transcriptional induction of these genes.

Microarray data also showed that when the expression of antimicrobial genes was induced, the expression of metabolic genes was reduced. How is this counter-regulation achieved? Mef2 was found to be phosphorylated on a conserved region of its DNA-binding domain in healthy D. melanogaster but this phosphorylation was lost following infection. Experiments using flies expressing wild-type non-phosphorylatable Mef2 or a constitutively phosphorylated form of Mef2 showed that only unphosphorylated forms of Mef2 associate with Tbp. So, the loss of Mef2 phosphorylation that occurs following infection facilitates the formation of the Mef2-Tbp complexes that are responsible for the induction of AMP expression. Consistent with the idea that phosphorylation switches Mef2 target gene repertoires, only the phosphorylatable forms of Mef2 promoted expression of metabolic genes.

So, in flies, phosphorylation of Mef2 controls a switch between ensuring storage of fat and glycogen and mounting a protective response to infection. 155, 435-447 (2013) 